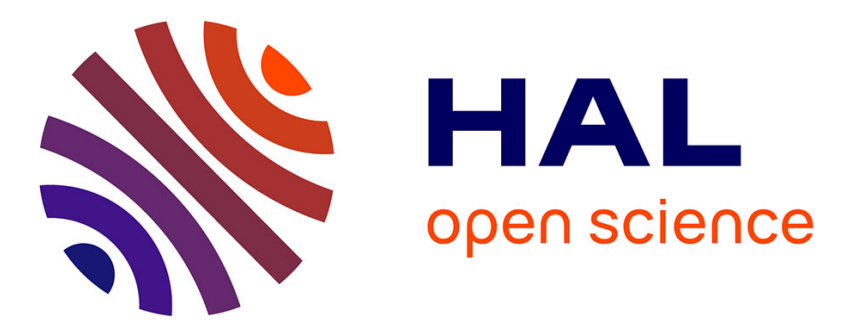

\title{
Towards Improving Students' Forum Posts Categorization in MOOCs and Impact on Performance Prediction
}

Fatima Harrak, Vanda Luengo, François Bouchet, Rémi Bachelet

\section{- To cite this version:}

Fatima Harrak, Vanda Luengo, François Bouchet, Rémi Bachelet. Towards Improving Students' Forum Posts Categorization in MOOCs and Impact on Performance Prediction. Learning @ Scale, Jun 2019, Chicago, United States. pp.47:1-47:4, 10.1145/3330430.3333661 . hal-02157333

\section{HAL Id: hal-02157333 \\ https://hal.science/hal-02157333}

Submitted on 21 Jun 2019

HAL is a multi-disciplinary open access archive for the deposit and dissemination of scientific research documents, whether they are published or not. The documents may come from teaching and research institutions in France or abroad, or from public or private research centers.
L'archive ouverte pluridisciplinaire HAL, est destinée au dépôt et à la diffusion de documents scientifiques de niveau recherche, publiés ou non, émanant des établissements d'enseignement et de recherche français ou étrangers, des laboratoires publics ou privés. 


\section{Towards Improving Students' Forum Posts Categorization in MOOCs and Impact on Performance Prediction}

Fatima Harrak

Sorbonne Université

CNRS, Laboratoire

d'Informatique de Paris 6

F-75005, Paris, France

fatima.harrak@lip6.fr

Vanda Luengo

Sorbonne Université

CNRS, Laboratoire

d'Informatique de Paris 6

F-75005, Paris, France

vanda.luengo@lip6.fr

\author{
François Bouchet \\ Sorbonne Université \\ CNRS, Laboratoire \\ d'Informatique de Paris 6 \\ F-75005, Paris, France \\ françois.bouchet@lip6.fr \\ Rémi Bachelet \\ Centrale Lille \\ University of Lille \\ France \\ remi.bachelet@ec-lille.fr
}

\begin{abstract}
Going beyond mere forum posts categorization is key to understand why some students struggle and eventually fail in MOOCs. We propose here an extension of a coding scheme and present the design of the associated automatic annotation tools to tag students' questions in their forum posts. Working of four sessions of the same MOOC, we cluster students' questions and show how the obtained clusters are consistent across all sessions and can be sometimes correlated with students' success in the MOOC. Moreover, it helps us better understand the nature of questions asked by successful vs. unsuccessful students.
\end{abstract}

\section{Author Keywords}

Student's question; discussion forum; coding scheme; clustering; student's performance; MOOC

\section{ACM Classification Keywords}

I.5.3 [Pattern recognition]: Clustering; K.3.1 [Computers and Education]: Computer Uses in Education

\section{Introduction}

Students' questions can be used to improve their learning experience and help teachers better understand their thinking. In MOOCs, discussion forums are a key feature and it has been shown that lack of social interaction as a valuable form of learning is one of the main concern [4]. Although 
several works have tried to show the impact of categoriz-

\begin{tabular}{lrrr} 
& N1 & N2 & N3 \\
\hline GDP5 & 17579 & 7655 & 2087 \\
GDP6 & 23315 & 10597 & 4717 \\
GDP7 & 19392 & 12224 & 3504 \\
GDP8 & 24603 & 14072 & 4760
\end{tabular}

Table 1: Descriptive statistics of the $4 \mathrm{MOOC}$ sessions considered (registration, messages and success)

\section{$\mathrm{N} 1$ = Students registered $\mathrm{N} 2=\mathrm{Nb}$ of posts}

$\mathrm{N} 3=\mathrm{Nb}$ of unique posters

\section{Kappa between the automatic} annotator and the manual annotation

$\kappa_{q}=0.91$

Question classification (DT)

$\kappa_{c}=0.66$

Course classification (SVM)

$\kappa_{0}=0.37$

dimension 0 classification (GBT)

$\kappa_{1}=0.68$

dimension 1 classification (GBT)

$\kappa_{2}=0.39$

dimension 2 classification (GBT)

$\kappa_{3}=0.56$

dimension 3 classification (GLM)

$\kappa_{4}=0.48$

dimension 4 classification (DT) ing students' posts [5], whether they are content-related [6, 2], urgent [1], they rarely look into the detailed content of the posts. We hypothesize that analyzing more finely the content of MOOC posts would help in particular to predict students' success.

We want to address two research questions: (RQ1) Can we reliably annotate questions extracted from MOOC forum posts according to a fine-grained multi-level coding scheme? And (RQ2) is there a consistent relationship between students' questions and their performance in the MOOC? We address RQ1 by extending an existing coding scheme and developing a coding tool using several classifiers in cascade to annotate sentences extracted from students' posts on a MOOC. Then we address RQ2 by using clustering to investigate whether the type of questions asked by students relate to their success.

\section{Research Context}

We consider log and forum data from four different sessions of the same French $\mathrm{MOOC}$ on project management called GDP (French acronym for project management) held in 2015 and 2016 (sessions 5 to 8). The forum works in a typical manner, organized around threads created by the pedagogical team to answer to technical or administrative issues, about homework or course content, among others. Table 1 provides some basic statistics on the forum usage and number of students registered. For each session we extracted the students' posts in course related topics, the final grade (out of 100) and whether students were successful or not (grade superior to $50 \%$ ).

\section{Question Coding Scheme}

Coding scheme design

We considered a sample of 500 messages from the 4 sessions randomly divided into 3 sub-samples $(200 / 100 / 200)$ to apply 3 successive categorization steps to define a coding scheme as proposed by Harrak et al. in another context of study [3]. The raw corpus contains messages posted in the discussion forum and can be very unstructured and noisy

(e.g. a message can contain several questions, opinions, answers to issues not course related, etc.). We excluded from this analysis: (1) messages coming from the instructors, (2) messages that are a reply to other ones (e.g. not the root messages), and (3) the threads which are explicitly not course related (e.g. a thread on technical issues).

The messages were segmented into several questions (using NLTK) and annotated according to their content. The course-based questions were annotated given the coding scheme from [3]. This coding scheme (cf. Table 2) consists in tagging each question according to 4 independent dimensions: a main mandatory one (dimension 1), and 3 optional ones (dimensions 2 to 4). For instance, a question could be a request to re-explain the way something work by providing another example (tagged as "Ree" on dimension 1, "Exa" on dimension 2, "Man" on dimension 3, and nothing (0) on dimension 4, i.e. [Ree,Exp,Man,0]). The noncourse related questions were then annotated according to dimension 0 (cf. Table 3). Two human annotators used as a unique reference the coding scheme introduced in Tables 2 and 3 to annotate each question. Their agreement was evaluated using Cohen's Kappa (average value: 0.71).

Automatic annotation

To annotate the entire corpus of messages, we performed the classical preprocessing steps on the training sample of 1307 segments (500 messages) manually annotated: to- 


\begin{tabular}{ll} 
Dim1 & Question type \\
\hline Ree & Re-explain / redefine \\
Dee & Deepen a concept \\
Ver & Validation / verification \\
\hline Dim2 & Explanation modality \\
& / Quest. subject \\
\hline Exp & Example \\
Sch & Schema \\
Cor & Correction \\
\hline Dim3 & Explanation type \\
\hline Def & Definition \\
Man & Manner (how?) \\
Rea & Reason (why?) \\
Rol & Roles (utility?) \\
Lin & Link between con- \\
& cepts \\
\hline Dim4 & Verification type \\
\hline Mis & Mistake / contradiction \\
Kno & Knowledge in course \\
Exp & Expected knowledge \\
& in assessment
\end{tabular}

Table 2: Coding scheme used to tag course-based students questions (adjusted from (Harrak et al. 2018)) kenization, stemming, punctuation removal (except for '?') and stopwords (non-meaningful words). We then counted the occurences of all the unigrams and bigrams. Each segment was represented by a binary word vector (' 1 ' if the word is in the segment, ' 0 ' otherwise). The number of keywords automatically extracted was reduced with feature selection to keep the most important and significant ones (removing less frequent and correlated unigrams / bigrams).

We then trained 3 stages of an automatic annotation tool to identify segments with questions, course vs. non-course related questions and the nature of those questions. Overall, 7 classifiers were then trained to annotate the corpus of segments respectively: (1) into question/non-question; (2) into course/non-course related questions (3) for non-course related questions, according to dim 0; (4-7) for coursebased questions, according to $\operatorname{dim} 1$ to 4 . For each classifier we trained 6 different models : Support Vector Machine (SVM), Generalized Linear Model (GLM), Gradient Boosted Trees (GBT), Decision Tree (DT), K-NN, Naive Bayes (NB) and Rule Induction (RI). All models were evaluated using 10 fold cross-validation on each of step.

\section{Links between questions and success}

To answer to RQ2, first we performed four clustering analyses using K-Means algorithm (with $\mathrm{k}$ varying between 2 and 10) over four datasets: students who asked questions in GDP5 ( $\mathrm{N}_{5}=278$ students), GDP6 ( $\left.\mathrm{N}_{6}=275\right)$, GDP7 $\left(N_{7}=314\right)$ and GDP8 $\left(N_{8}=287\right)$. We performed the clustering using as features for each student the proportion of each question asked in each dimension (e.g. the proportion of questions with value "App" in dimension 1) asked overall. The results reveal that two similar clusters are found in each session of the MOOC, called C1 and C2.

The second step consisted in characterizing the clusters by

\begin{tabular}{ll} 
Dim0 & Categories \\
\hline Soc & Socialization \\
Adm & Administrative issues \\
Exa & Exam/ quiz modality \\
Tec & Technical issues \\
Res & Ressources not found \\
Too & Tools \\
Pha & Phatic (has no real value)
\end{tabular}

Table 3: Coding scheme created (translated from French) from manual annotation to tag non-course related students' questions

analyzing which of the 19 dimensions used to extract them differ significantly. We ran 76 (19 times 4) Mann-Whitney

$U$ tests for each dimension for each of the 4 sessions, and adjusted the threshold $p$-value with Bonferroni correction (adjusted $p$-value $=.0007$ ). Table 4 summarizes the results for the dimensions with a statistically significant difference in at least one of the four sessions. When compared to $\mathrm{C} 2$, students in $\mathrm{C} 1$ always ask more questions about the exams, less verification questions in particular about concepts from the course. They sometimes also ask more administrative questions (GDP5 and 6), less questions linking two concepts (GDP7) and about how to proceed (GDP8) as well as less questions to deepen their understanding (GDP8).

The third step consisted in characterizing the clusters in terms of attributes not used for the clustering. We ran 4 Chi-square tests for the success and 4 Mann-Whitney $U$ for the final grade. Those tests revealed a statistically significant difference for the final grade for sessions 6 and 8 only ( $p=.014$ and .040 and $\eta^{2}=0.018$ and 0.010 respectively), with a higher final score for $\mathrm{C} 2$, and a higher proportion of students from C2 who obtain their certificate at the end for session 8 only ( $\chi^{2}=6.77, p=.009,79.9 \%$ vs. $65.5 \%$ ). 


\begin{tabular}{|c|c|c|c|c|c|c|c|c|c|c|c|c|c|c|c|c|c|c|c|c|c|c|c|c|c|c|c|}
\hline \multirow[b]{2}{*}{ Cluster } & \multirow[b]{2}{*}{$N$} & \multicolumn{3}{|c|}{$\operatorname{dim}_{e x a}$} & \multicolumn{3}{|c|}{$\operatorname{dim}_{a d m}$} & \multicolumn{3}{|c|}{$\operatorname{dim} \mathbf{1}_{v e r}$} & \multicolumn{3}{|c|}{$\operatorname{dim} \mathbf{1}_{d e e}$} & \multicolumn{3}{|c|}{$\operatorname{dim} \mathbf{3}_{\operatorname{man}}$} & \multicolumn{3}{|c|}{$\operatorname{dim} 3_{l i n}$} & \multicolumn{3}{|c|}{$\operatorname{dim} \mathbf{4}_{c o n}$} & \multicolumn{3}{|c|}{ F. Grade } & \multirow{2}{*}{$\begin{array}{l}\text { Suc. } \\
\text { Prop }\end{array}$} & \multirow{2}{*}{$\frac{\text { Course }}{\text { Prop }}$} \\
\hline & & Q1 & $M d$ & Q3 & Q1 & $M d$ & Q3 & Q1 & $M d$ & Q3 & Q1 & Md & Q3 & Q1 & $M d$ & Q3 & Q1 & $M d$ & Q3 & Q1 & $M d$ & Q3 & Q1 & $M d$ & Q3 & & \\
\hline$C 1_{G D P 5}$ & 189 & 0 & $0.25^{*}$ & 1 & 0 & $0^{*}$ & 0.33 & 0 & $0^{*}$ & 0 & 0 & 0 & 0.18 & 0 & 0 & 0 & 0 & 0 & 0 & 0 & $0^{*}$ & 0 & 47.2 & 66.3 & 90.8 & 0.74 & $0.20^{\star}$ \\
\hline$C 2_{G D P 5}$ & 96 & 0 & $0^{*}$ & 0 & 0 & $0^{*}$ & 0 & 0 & $0.93^{*}$ & 1 & 0 & 0 & 0.12 & 0 & 0 & 0 & 0 & 0 & 0 & 0.5 & $0.67^{*}$ & 1 & 52.82 & 282.4 & 92.22 & 0.78 & $0.86^{*}$ \\
\hline$C 1_{G D P 6}$ & 177 & 0 & $0.33^{*}$ & 1 & 0 & $0^{*}$ & 0.17 & 0 & $0^{*}$ & 0 & 0 & 0 & 0.14 & 0 & 0 & 0 & 0 & 0 & 0 & 0 & $0^{*}$ & 0 & 46.77 & $756.47^{*}$ & 89.67 & 0.70 & $0.22^{*}$ \\
\hline$C 2_{G D P 6}$ & 98 & 0 & $0^{*}$ & 0 & 0 & $0^{*}$ & 0 & 0.50 & $1^{*}$ & 1 & 0 & 0 & 0 & 0 & 0 & 0 & 0 & 0 & 0 & 0.50 & $0.75^{\star}$ & 1 & 51.88 & 8 84.61* & 93.64 & 0.78 & $0.86^{*}$ \\
\hline$C 1_{G D P 7}$ & 189 & 0 & $0.33^{*}$ & 1 & 0 & 0 & 0 & 0 & $0^{*}$ & 0 & 0 & 0 & 0.33 & 0 & 0 & 0 & 0 & $0^{*}$ & 0 & 0 & $0^{*}$ & 0 & 79.80 & 91.15 & 94.70 & 0.80 & $0.28^{*}$ \\
\hline$C 2_{G D P 7}$ & 125 & 0 & $0^{*}$ & 0.15 & 0 & 0 & 0 & 0.50 & $0.70^{*}$ & 1 & 0 & 0 & 0.17 & 0 & 0 & 0 & 0 & $0^{*}$ & 0 & 0.50 & $0.67^{*}$ & 1 & 81.72 & 291.92 & 96.20 & 0.81 & $0.84^{*}$ \\
\hline$C 1_{G D P 8}$ & 88 & 0.67 & $1^{*}$ & 1 & 0 & 0 & 0 & 0 & $0^{*}$ & 0 & 0 & $0^{*}$ & 0 & 0 & $0^{*}$ & 0 & 0 & 0 & 0 & 0 & $0^{*}$ & 0 & 33.30 & $84.68^{*}$ & 91.93 & $0.66^{*}$ & $0.08^{*}$ \\
\hline$C 2_{G D P 8}$ & 199 & 0 & $0^{*}$ & 0 & 0 & 0 & 0 & 0 & $0.44^{*}$ & 1 & 0 & $0^{*}$ & 0.33 & 0 & $0^{*}$ & 0 & 0 & 0 & 0 & 0 & $0.33^{*}$ & 1 & 77.90 & $88.33^{*}$ & 92.61 & $0.80^{*}$ & $0.66^{*}$ \\
\hline
\end{tabular}

Table 4: Summary of median, first and third quartiles of the variables used for the clustering ( ${ }^{*}$ means significant with Bonferroni correction) and for the dependent variables (final grade, proportion of success and proportion of course questions) for each cluster and for each course

We also see that the proportion of course questions is significantly higher for $\mathrm{C} 2$ for each session.

\section{Discussion and Conclusion}

We have presented a tool to annotate MOOC posts in a more fine-grained manner than usual approaches. When annotating posts across different sessions of a same MOOC, consistent clusters of questions appeared, which are sometimes correlated with the performance. Although course vs. non-course may be enough to help in success prediction, our approach offers a better understanding of the nature of questions from successful vs. unsuccessful students, opening the path to a finer interpretation of what some students are doing wrong. We envision to replicate this analysis on other MOOCs to see if similar patterns can be found.

\section{REFERENCES}

1. Omaima Almatrafi, Aditya Johri, and Huzefa Rangwala. 2018. Needle in a haystack: Identifying learner posts that require urgent response in MOOC discussion forums. Computers \& Education 118 (March 2018), 1-9.
2. Yi Cui and Alyssa Friend Wise. 2015. Identifying Content-Related Threads in MOOC Discussion Forums. In Proc. of the Second (2015) ACM Conf. on Learning@ Scale L@S '15. ACM Press, Vancouver, BC, Canada, 299-303.

3. Fatima Harrak, François Bouchet, Vanda Luengo, and Pierre Gillois. 2018. Profiling Students from Their Questions in a Blended Learning Environment. In Proc. of the 8th International Conf. on Learning Analytics and Knowledge (LAK '18). ACM, New York, NY, USA, 102-110.

4. Carolyn Penstein Rosé and Oliver Ferschke. 2016. Technology Support for Discussion Based Learning: From Computer Supported Collaborative Learning to the Future of Massive Open Online Courses. International J. of Artificial Intelligence in Education 26, 2 (June 2016), 660-678.

5. Glenda S Stump, Jennifer DeBoer, Jonathan Whittinghill, and Lori Breslow. 2013. Development of a Framework to Classify MOOC Discussion Forum Posts: Methodology and Challenges. (2013), 20

6. Alyssa Friend Wise and Yi Cui. 2018. Learning communities in the crowd: Characteristics of content related interactions and social relationships in MOOC discussion forums. Computers \& Education 122 (July 2018), 221-242. 